\title{
A Technique for Transient Suppression of IIR Notch Filter
}

\author{
Soo-Chang Pei and Chien-Cheng Tseng \\ Department of Electrical Engineering \\ National Taiwan University \\ Taipęi, Taiwan, R.O.C.
}

\begin{abstract}
In this paper, a technique for suppressing the transient states of IIR notch filters is presented. This technique mainly uses the vector projection to find the good initialization of a notch filter. When a notch filter is used to cancel $60 \mathrm{~Hz}$ interference in the recording of electrocardiograms (ECG), the performance of the notch filter with transient suppression is better than that of the notch filter with arbitrary initialization. The improvements realized by this technique is at the cost of little additional computational load at the beginning of filtering.
\end{abstract}

\section{Introduction}

The IIR notch filters are very useful in many signal processing applications such as the retrieval of sinusoids in noise, eliminating the sinusoidal power line disturbances in a measurement signal [1]-[5]. One typical example is to cancel $60 \mathrm{~Hz}$ power line interference in the recording of electrocardiogram (ECG) [1]. The other example is to eliminate a portion of the feedback signal in a flexible missible system to keep the system from destroying itself [2][3].

The problem tackled in this paper is motivated by the work which we use notch filters to remove $60 \mathrm{~Hz}$ interference in ECG signal. When we pass a corrupted signal through the IIR notch filter to remove the sinusoidal interference, the transient response distorts the filter output on the startup. Typically, a notch filter with the narrower $3 \mathrm{~dB}$ rejection bandwidth has the longer transient response at the filter output. However, we usually prefer a notch filter with narrower $3 \mathrm{~dB}$ rejection bandwidth to faithly seperate sinusoidal component and broadband component. Hence, a trade-off between the duration of transient and the distortion of seperateness must be considered. In this paper, a technique is proposed to suppress transient response.

The technique proposed in this paper is based on the following two steps. First, we use vector projection to decompose the first $M$ samples of input signal into the sinusoidal interference component and the ECG signal component. Next, we use ECG signal component as an initial of the notch filter to perform filtering operation. Consequently, tansient response is reduced dramatically. Although additional computation cost is required on the startup of filtering, it is small and not a problem for real time applications.

\section{Transient State in Notch Filter}

In the section, the transient response of notch filter will be studied. The transfer function of second order IIR notch filter is given by [3]

$$
Y(z)=\frac{1}{2} \frac{\left(1+a_{2}\right)-2 a_{1} z^{-1}+\left(1+a_{2}\right) z^{-2}}{1-a_{1} z^{-1}+a_{2} z^{-2}} X(z)
$$

where $X(z)$ and $Y(z)$ are $z$ transforms of notch filter input $x(n)$ and output $y(n)$, respectively. The notch frequency $\omega_{0}$ and $3 \mathrm{~dB}$ rejection bandwidth $\Omega$ relates to the filter coefficients $a_{1}$ and $a_{2}$ by the following expression

$$
a_{i}=\frac{2 \cos \left(w_{0}\right)}{1+\tan \left(\frac{\Omega}{2}\right)}
$$




$$
a_{2}=\frac{1-\tan \left(\frac{\Omega}{2}\right)}{1+\tan \left(\frac{\Omega}{2}\right)}
$$

In this paper, the input signal $x(n)$ of notch filter is a sinusoidal interference $d(n)$ with frequency $\omega_{0} \mathrm{em}$ bedded in a ECG signal $s(n)$, i.e.

$$
x(n)=s(n)+d(n)
$$

where $d(n)$ is given by

$$
d(n)=A_{0} \sin \left(\omega_{0} n+\phi\right)
$$

When $3 \mathrm{~dB}$ rejection bandwidth $\Omega$ is very small, the notch filter output $y(n)$ is almost same as $s(n)$. Now, we summarize the notch filtering operation with arbitrary initial as follows:

Filtering 1: Given signal $x(n)$ and $\omega_{0}, \Omega$

Step 1. Calculate filter coefficient $a_{1}$ and $a_{2}$.

Step 2. Choose arbitrary initial $y(-1)$ and $y(-2)$.

Step 3. For $n=0$ to $\mathrm{N}$, calculate the output $y(n)$ by notch filter.

As an example, we use Filtering 1 to remove the $60 \mathrm{~Hz}$ power line interference in the recording of ECG signal. The samples used in this example have 8 bit$\mathrm{s}$ and the sampling rate is $800 \mathrm{~Hz}$. Fig.1(a) shows an ECG waveform with a excessive amount of $60 \mathrm{~Hz}$ interference. Fig.1(b)-(c) show the outputs of Filtering 1 with various $3 \mathrm{~dB}$ notch rejection bandwidth $\Omega$ and zero initial $y(-1), y(-2)$. From these results, it is clear that the severe transient states appear in the beginning of notch filter output. Also, the narrower bandwidth $\Omega$ the notch filter has, the longer duration of transient states the notch output has. Because we usually prefer the bandwidth $\Omega$ is very narrow for reducing the distortion of ECG signal, a technique for transient states suppression must be developed. In next section, we will propose a suppression technique to achieve this purpose.

\section{Notch Filtering with Transient State Suppression}

In this section, we will develop a suppression technique so as to the transient states of notch filter output can be removed. The basic procedures of this technique can be described as follows.

At the beginning, we arrange first $M$ input signal samples into the following $M \times 1$ data vector

$$
X=[x(0) \quad x(1) \quad x(2) \cdots x(M-1)]^{t}
$$

From eq(3), this vector can be rewritten as

$$
X=S+D
$$

where vectors $S$ and $D$ are

$$
\begin{aligned}
S & =\left[\begin{array}{lll}
s(0) & s(1) & s(2) \cdots s(M-1)
\end{array}\right]^{t} \\
D & =\left[\begin{array}{lll}
d(0) & d(1) & d(2) \cdots d(M-1)
\end{array}\right]^{t}
\end{aligned}
$$

If we define matrix $A$ as

$$
A=\left[\begin{array}{ccccc}
1 & \cos \left(\omega_{0}\right) & \cos \left(2 \omega_{0}\right) & \cdots & \cos \left((M-1) \omega_{0}\right) \\
0 & \sin \left(\omega_{0}\right) & \sin \left(2 \omega_{0}\right) & \cdots & \sin \left((M-1) \omega_{0}\right)
\end{array}\right]^{t}
$$

then vector $D$ is exact in the column space of matrix $A[6]$. Thus, we can use projection operation to decompose $X$ into sinusiodal part $D$ and signal part $S$. If we define projection operator $P$ as

$$
P=A\left(A^{t} A\right)^{-1} A^{t}
$$

then sinusoidal part can be estimated by

$$
\hat{D}=P X \text {. }
$$

Moreover, signal part can be obtained by

$$
\begin{aligned}
\hat{S} & =X-\hat{D} \\
& =(I-P) X
\end{aligned}
$$

where $I$ is identity matrix. Instead of using arbitrary initial in Filtering 1, we use $\hat{S}$ as an initial to perform filtering operation. We summarize this new filtering process as follows:

Filtering 2: Given signal $x(n)$ and $\omega_{0}, \Omega, M$

Step 1. Calculate filter coefficient $a_{1}$ and $a_{2}$.

Step 2. Construct input data vector $X$ and projection matrix $P$.

Step 3. Calculate first $M$ output samples $y(n)$ by $(I-P) X$.

Step 4. For $n=M+1$ to $N$, calculate output samples $y(n)$ by notch filter.

Now, the preceding example in Fig.1 is used to test this new filtering technique. The scenario of the experiment are all the same. Fig.2(a) and (b) show the outputs of notch filter with rejection bandwidth $\Omega=0.4 \mathrm{~Hz}$ and $\Omega=0.8 \mathrm{~Hz}$. The number of input samples $M$ is chosen as 10 . From this result, it is clear that the transient states are almost removed by our propsed technique. 
Remark 1: In the above, we have not stated how to choose $M$. In our experience, when $M$ is chosen as an integer form 5 to 15 , the Filtering 2 will perform very well.

Remark 2: In general, the second order notch filter can only remove one sinusoidal interference. When signal is corrupted by harmonic interference, then high order notch filter must be used. In this case, the proposed suppression technique can be extended directly. Without loss generality, we investigate a six order notch filter as follows. The transfer function considered is given by [7]

$$
H(z)=\frac{Q(z)}{Q(\rho z)}
$$

where

$Q(z)=1+a_{1} z^{-1}+a_{2} z^{-2}+a_{3} z^{-3}+a_{2} z^{-4}+a_{1} z^{-5}+z^{-6}$

Usually, the zeros of $Q(z)$ are all on the unit circle and denoted by $e^{ \pm j \theta_{i}}(i=1,2,3)$. The notch frequencies and $3 \mathrm{~dB}$ rejection bandwith are given by

$$
\begin{aligned}
w_{i} & =\theta_{i} \quad i=1,2,3 \\
\Omega & =\pi(1-\rho)
\end{aligned}
$$

Moreover, the input signal have the form

$$
x(n)=s(n)+\sum_{i=1}^{3} A_{i} \sin \left(\omega_{i} n+\phi_{i}\right)
$$

As second order notch filter, transient states appear in notch filter output if arbitrary initial is used. Now, we summarize a technique to suppress tranisent below.

Filtering 3: Given signal $x(n)$ and $\Omega, M, \omega_{i}(\mathrm{i}=1,2,3)$

Step 1. Calculate filter parameters $\rho$, and $a_{i}(i=1,2,3)$

Step 2. Construct input data vector $X$ and matrix $A$.

$A=\left[\begin{array}{ccccc}1 & \cos \left(\omega_{1}\right) & \cos \left(2 \omega_{1}\right) & \ldots & \cos \left((M-1) \omega_{1}\right) \\ 0 & \sin \left(\omega_{1}\right) & \sin \left(2 \omega_{1}\right) & \ldots & \sin \left((M-1) \omega_{1}\right) \\ 1 & \cos \left(\omega_{2}\right) & \cos \left(2 \omega_{2}\right) & \ldots & \cos \left((M-1) \omega_{2}\right) \\ 0 & \sin \left(\omega_{2}\right) & \sin \left(2 \omega_{2}\right) & \ldots & \sin \left((M-1) \omega_{2}\right) \\ 1 & \cos \left(\omega_{3}\right) & \cos \left(2 \omega_{3}\right) & \ldots & \cos \left((M-1) \omega_{3}\right) \\ 0 & \sin \left(\omega_{3}\right) & \sin \left(2 \omega_{3}\right) & \ldots & \sin \left((M-1) \omega_{3}\right)\end{array}\right]$

Step 3. Calculate projection matrix $P=A\left(A^{t} A\right)^{-1} A^{t}$. Step 4. Calculate first $M$ output samples $y(n)$ by $(I-P) X$.

Step 5. For $n=M+1$ to $N$, calculate output samples $y(n)$ by notch filter.
We have used various examples to test this new filtering technique. All results reveal that the transient states are almost removed by our proposed technique in high order notch filter case.

\section{Conclusion}

This paper presents a technique to suppress transient states of IIR notch filters. Simulation examples have been shown that this technique can improve the performance of $60 \mathrm{~Hz}$ interference canceling application if proper computation load increases. In addition, it will be interesting to extend this technique to the transiet suppression of the lowpass and bandpass filters. This topic will be studied in the future.

\section{References}

[1] J.C.Huhta and J.G.Webster, "60-Hz interference in Electrocadiograph", IEEE Trans. Bio-Med. Eng., Vol. BEM-20, pp.91-101, March 1973.

[2] R.Carney, "Design of a digital notch filter with tracking requirements", IEEE Trans. Space Electron. Telem. Vol. SET-9, pp.109-114, Dec.1963.

[3] K.Hirano, S.Nishimura, and S.K.Mitra, "Design of digital notch filters", IEEE Trans. Circuit and Syst., Vol. CAS-21, pp. 540-546, July 1974.

[4] M.H.Er, "Designing notch filter with controlled null width", Signal Processing, Vol.24, No.3, pp.319-329, Sep. 1991

[5] G.W.Medlin, "A novel design technique for tuneable notch filters" ,Int'l conf. on circuits and systems, New Orleans, Louisiana, U.S.A. pp471-474, May 1990.

t [6] G. Strang, "Linear Algebra and Its Application", 2nd ed., Academic Press, N.Y., 1980

[7] A.Nehorai and B.Porat, "Adaptive comb filtering for harmonic signal enhancement", IEEE Trans. Acoust. Speech, Signal processing, Vol. ASSP-34 pp.1124-1138 Vol. 1986. 


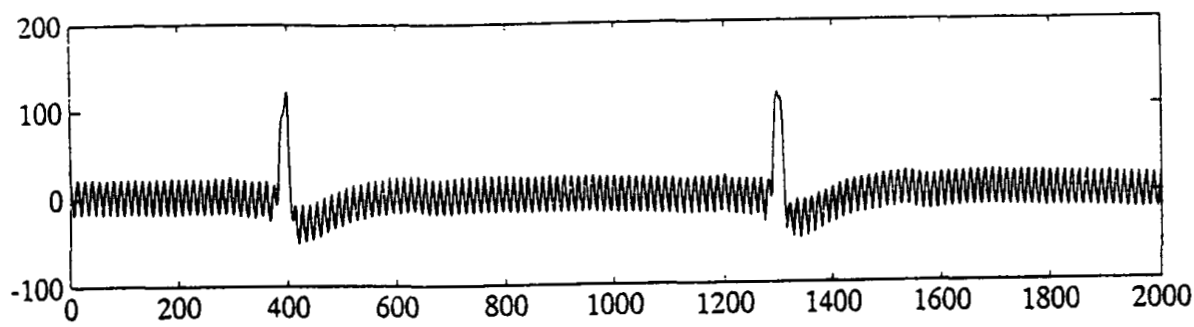

(a)

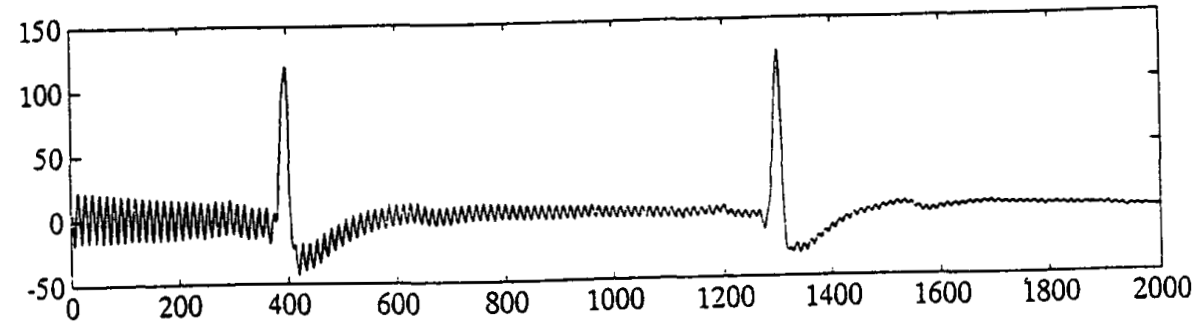

(b)

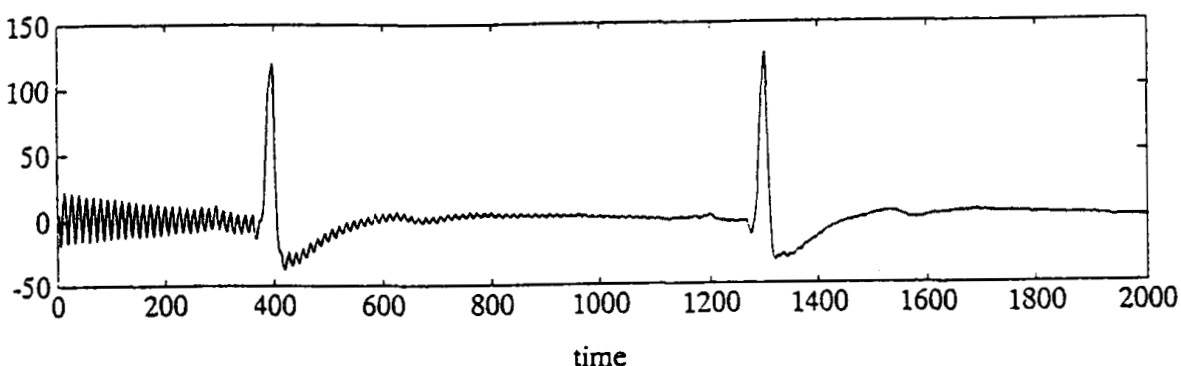

(c)

Fig.1: (a) Notch filter input. (b)Notch filter output with $y(-1)=y(-2)=0$ and $\Omega=0.4 \mathrm{~Hz}$. (c)Notch filter output with $y(-1)=y(-2)=0$ and $\Omega=0.8 \mathrm{~Hz}$.

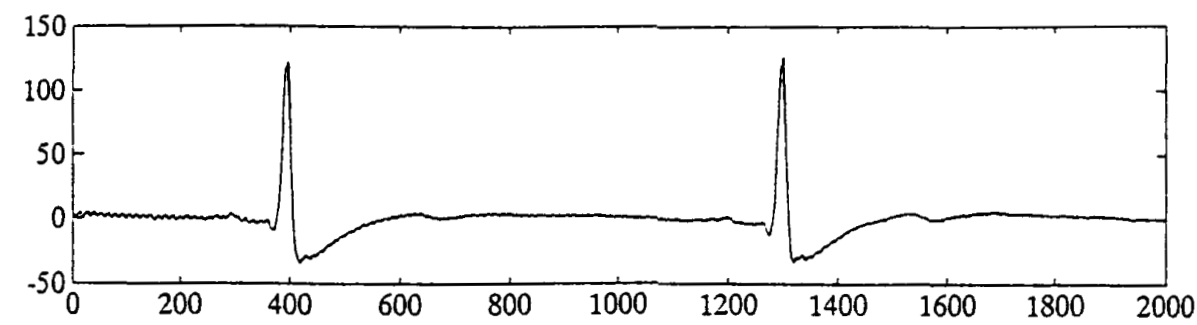

(a)

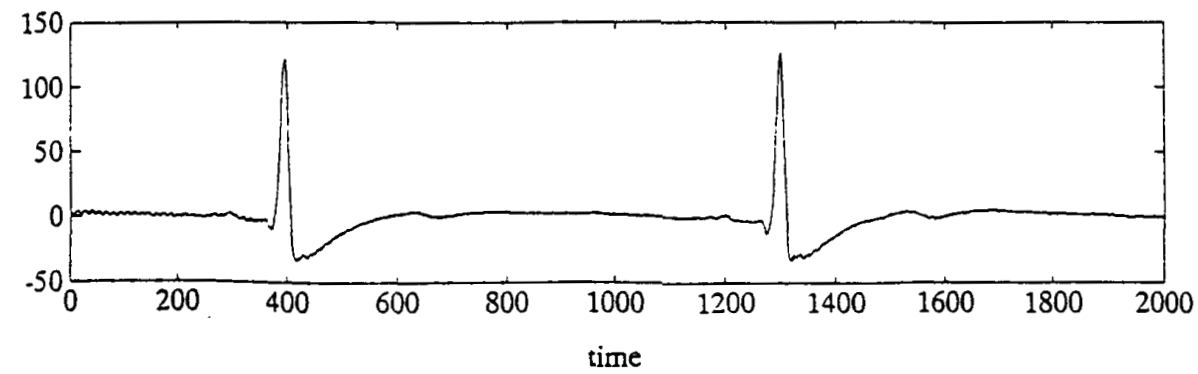

(b) Fig.2: Notch filter outputs with transient suppression. (a) $\Omega=0.4 \mathrm{~Hz}$ and $M=10$. (b) $\Omega=$
$0.8 \mathrm{~Hz}$ and $M=10$.

III-212 